\title{
High incidence of leukemia in large animals after stem cell gene therapy with a HOXB4-expressing retroviral vector
}

\author{
Xiao-Bing Zhang, ${ }^{1}$ Brian C. Beard,1 ${ }^{1}$ Grant D. Trobridge,,${ }^{1,2}$ Brent L. Wood, ${ }^{3}$ George E. Sale, ${ }^{1}$ \\ Reeteka Sud, ${ }^{1}$ R. Keith Humphries, ${ }^{4}$ and Hans-Peter Kiem ${ }^{1,2}$

\begin{abstract}
${ }^{1}$ Clinical Research Division, Fred Hutchinson Cancer Research Center, Seattle, Washington, USA. ${ }^{2}$ Departments of Medicine and Pathology and
\end{abstract} \\ ${ }^{3}$ Department of Laboratory Medicine, University of Washington, Seattle, Washington, USA. ${ }^{4}$ Terry Fox Laboratory, British Columbia Cancer Agency, \\ and Department of Medicine, University of British Columbia, Vancouver, British Columbia, Canada.
}

\begin{abstract}
Retroviral vector-mediated HSC gene therapy has been used to treat individuals with a number of life-threatening diseases. However, some patients with SCID-X1 developed retroviral vector-mediated leukemia after treatment. The selective growth advantage of gene-modified cells in patients with SCID-X1 suggests that the transgene may have played a role in leukemogenesis. Here we report that 2 of 2 dogs and 1 of 2 macaques developed myeloid leukemia approximately 2 years after being transplanted with cells that overexpressed homeobox B4 (HOXB4) and cells transduced with a control gammaretroviral vector that did not express HOXB4. The leukemic cells had dysregulated expression of oncogenes, a block in myeloid differentiation, and overexpression of HOXB4. HOXB4 knockdown restored differentiation in leukemic cells, suggesting involvement of HOXB4. In contrast, leukemia did not arise from the cells carrying the control gammaretroviral vector. In addition, leukemia did not arise in 5 animals with high-level marking and polyclonal long-term repopulation following transplantation with cells transduced with an identical gammaretrovirus vector backbone expressing methylguanine methyltransferase. These findings, combined with the absence of leukemia in many other large animals transplanted with cells transduced with gammaretroviral vectors expressing genes other than HOXB4, show that HOXB4 overexpression poses a significant risk of leukemogenesis. Our data thus suggest the continued need for caution in genetic manipulation of repopulating cells, particularly when the transgene might impart an intrinsic growth advantage.
\end{abstract}

\section{Introduction}

Gene therapy holds great promise for providing new treatments for a large number of genetic and acquired diseases (1), and retroviruses are currently the most efficient vectors for gene delivery to HSCs. Gammaretroviral vectors have shown early clinical success for X-linked SCID (SCID-X1) (2-5), adenosine deaminase SCID (ADA-SCID) (6-8), and X-linked chronic granulomatous disease (X-CGD) (9). However, 4 SCID-X1 patients developed T cell leukemia after gene transfer of IL2R $\gamma$ via an MLV-based gammaretroviral vector into HSCs $2-3$ years after treatment, but not in other gene therapy trials $(10,11)$. These findings led to the notion that leukemogenesis caused by retroviral vectors may be associated with particular transgenes.

Studies have been carried out in mouse models to address safety issues involved in retroviral vector-based stem cell gene therapy (12-14). However, findings in murine models may not always clearly predict outcomes in a clinical setting, likely because of intrinsic differences between humans and mice. In addition, much higher stem cell doses are typically employed in clinical trials, resulting in the infusion of significantly higher numbers of gene-modified stem cells compared with mouse studies. In concert with this, studies in large animals like dogs and nonhuman primates have

Nonstandard abbreviations used: HOXB4, homeobox B4; MGMT, methylguanine methyltransferase; $\mathrm{MSCV}$, murine stem cell virus; PRDM16, PR domain containing 16 ; TSS, transcription start site; YFP, yellow fluorescent protein.

Conflict of interest: The authors have declared that no conflict of interest exists. Citation for this article: J. Clin. Invest. 118:1502-1510 (2008). doi:10.1172/JCI34371. been more predictive than mouse studies. Thus, we have used large animal models to study HSC gene transfer to address the safety of gene-modified HSCs. We have reported that long-term analysis of large animals (17 dogs and 23 baboons) that received gene-modified cells identified no vector-mediated malignancies (15). More than 40 animals have now been followed a median time of 3.1 years and have maintained normal hematopoiesis.

Accumulating evidence from mouse studies suggests that homeobox B4 (HOXB4) overexpression enhances in vivo $(16,17)$ and ex vivo expansion of HSCs $(18,19)$. In contrast to the strong leukemogenic capacity of other members of the HOX gene clusters (20), HOXB4expanded HSCs retain their normal differentiation and long-term repopulation potential, and no hematologic abnormalities have been detected in large groups of mice that were transplanted with HOXB4-transduced HSCs $(16,18)$. Our previous study in nonhuman primates showed that HOXB4 overexpression significantly expands repopulating cells, in particular short-term repopulating cells (21).

Here we characterized the leukemias that developed in the 3 animals that received HOXB4-transduced $\mathrm{CD} 34^{+}$cells approximately 2 years after transplantation. We compared the findings to a control group of animals that received transduced CD $34^{+}$cells with the same vector backbone but expressing the methylguanine methyltransferase (MGMT) gene.

\section{Results}

Development of leukemia in 3 animals approximately 2 years after transplantation. We investigated the effects of HOXB4 overexpression in the dog and nonhuman primate models. Our studies show- 

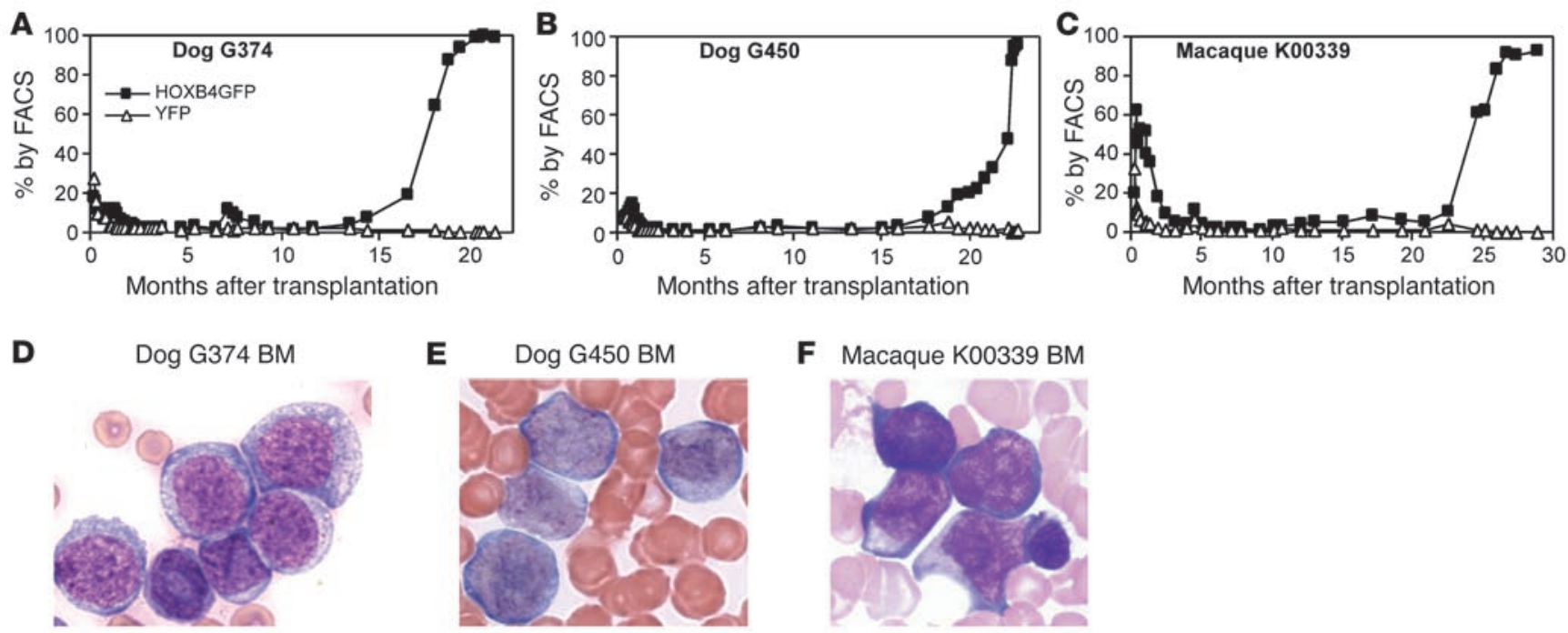

F Macaque K00339 BM
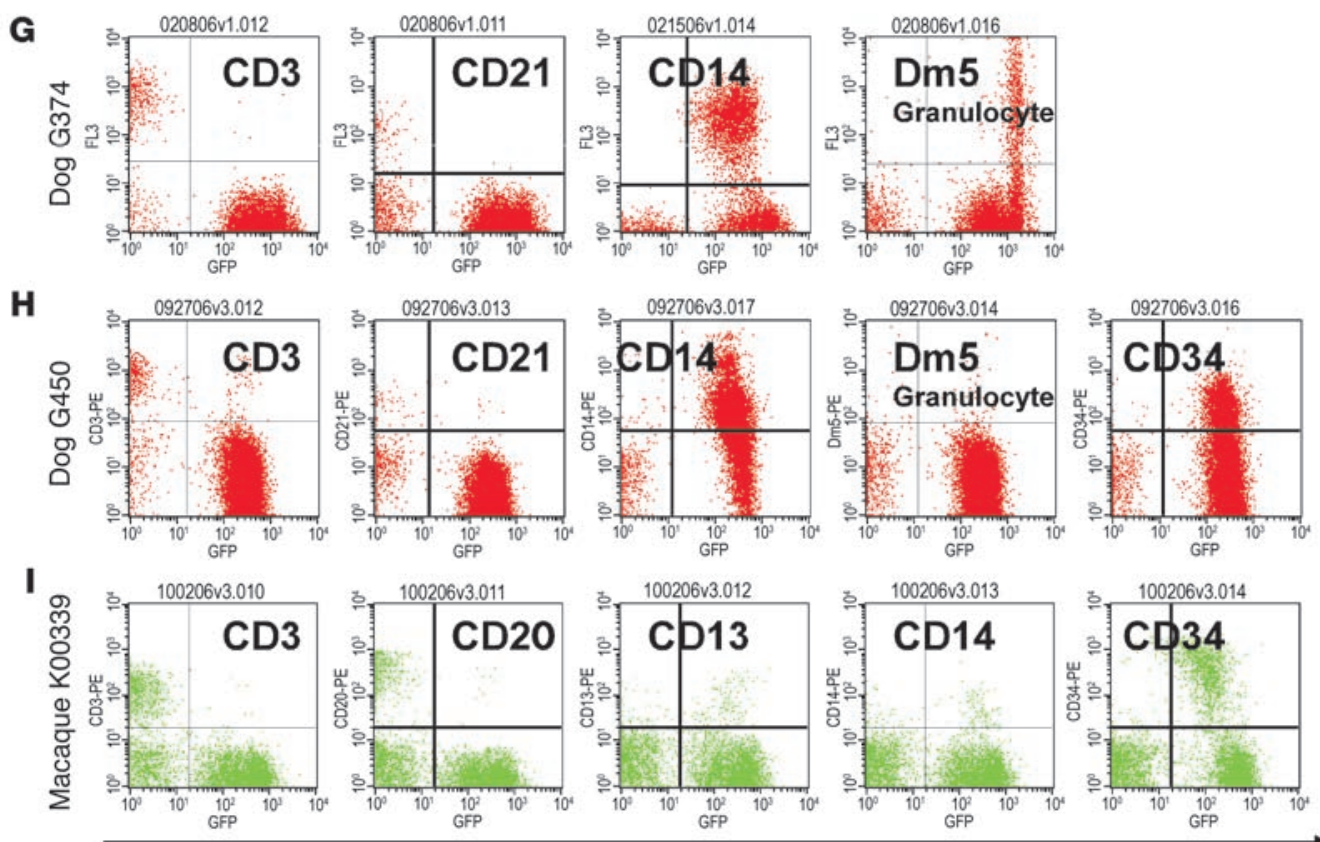

HOXB4GFP

Figure 1

Development of leukemia in 2 dogs and 1 macaque transplanted with HOXB4-overexpressing CD34+ cells. Marking levels in granulocytes in dogs G374 (A) and G450 (B) and macaque K00339 (C). Representative marrow morphology at necropsy for G374 (D), G450 (E), and K00339 (F). High numbers of blast cells were observed in the marrow. Subset analysis of marrow samples from G374 (G), G450 (H) and K00339 (I). After red blood cell lysis, nucleated marrow cells were stained with antibodies to the T cell marker CD3, B cell marker CD20 or CD21, monocyte marker CD14, granulocyte marker Dm5 or CD13, and stem/progenitor cell marker CD34.

ing the effect of HOXB4 on hematopoietic repopulating cells in nonhuman primates have been previously published (21). Similar to our findings in nonhuman primates, we observed higher levels of HOXB4-overexpressing cells early after transplantation compared with levels after 1 month in dogs (Supplemental Figure 1; supplemental material available online with this article; doi:10.1172/JCI34371DS1). One of the 3 dogs that received HOXB4-transduced CD $34^{+}$cells died 3 months after transplantation due to transplantation-related complications. The other 2 dogs (G374 and G450) were followed for 2 years. Approximate- ly 15 months after transplantation, we observed a spontaneous increase of HOXB4-marked cells in dogs G374 and G450, while control yellow fluorescent protein-marked (YFP-marked) cells gradually decreased to $<0.1 \%$ (Figure $1, \mathrm{~A}$ and $\mathrm{B}$ ). The same pattern was seen in macaque K00339 at 22 months after transplantation (Figure 1C).

Dog G374 developed an increased white blood cell count with an increase in monocytes and a decrease in platelet count (Supplemental Figure 2). Bone marrow analysis confirmed the diagnosis of acute myelomonocytic leukemia (Figure 1D). Dog G450 
A

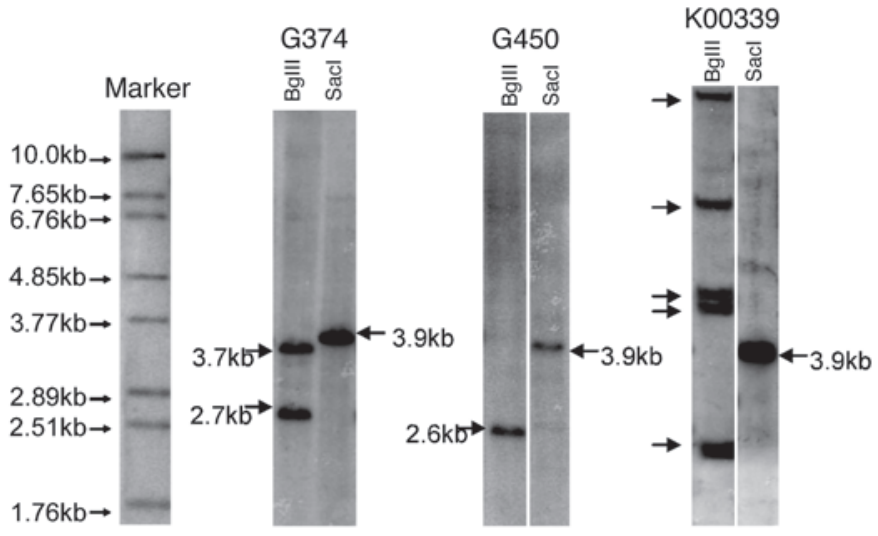

B

G374
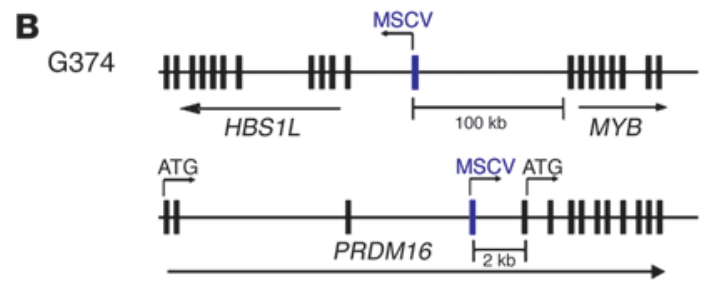

C

G450

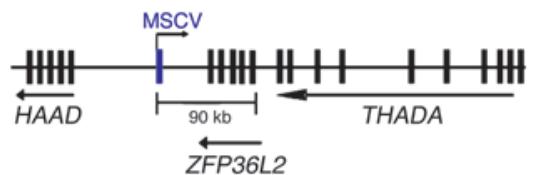

D
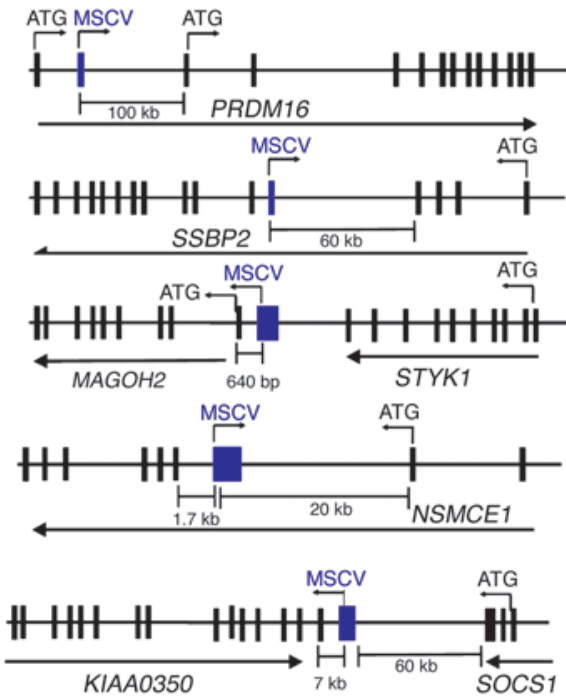

Figure 2

Retroviral integration induced mutagenesis is associated with leukemogenesis. (A) Southern blot analyses of marrow samples from G374, G450, and K00339 demonstrate monoclonality. Marrow DNA was digested with Bglll, which cuts the transgene once, releasing a unique band for each integrant. Digestion with Sacl, which cuts the transgene twice, showed a 3.9-kb band for all the integrants. (B-D) Integration sites were determined by LAM-PCR, and the schematic representations of integration sites for G374 (B), G450 (C), and K00339 (D) are shown. Exons are represented by black boxes. MSCV indicates the integration site, and the arrow indicates the orientation. ATG denotes the translation start site. Note that exons and introns are not to scale. (E-G) Dysregulated expression of genes in close vicinity of the integration sites for G374 (E), G450 (F), and K00339 (G). SYBR Green real-time RT-PCR was performed to determine the expression levels of dog MYB (E), dog ZFP36L2 and thyroid adenoma associated (THADA) (F), and macaque PRDM16, SSBP2, and SOCS1 (G). Canine PRDM16 (E) and macaque MAGOH2 (G) expression was undetectable in samples from normal control animals. (G) Expression of NSMCE1 was undetectable in K00339 and control animals. Marrow mRNA samples from normal animals were used as controls. M, DNA ladder.

developed pancytopenia and an increased number of leukemic blast cells in the peripheral blood 20 months after transplantation (Supplemental Figure 3). Bone marrow and peripheral blood analysis confirmed the development of acute myeloid leukemia (AML) (Figure 1E).

Twenty-five months after transplantation, macaque K00339 developed a decreased hematocrit, an increase in nucleated red blood cells, and eventually an increase in white blood cells with circulating blasts (Supplemental Figure 4). Marrow analysis confirmed AML (Figure 1F).

All the animals were euthanized due to deteriorating physical condition associated with leukemia. Pathological examination of bone marrow and other tissues confirmed the diagnoses of leukemia. Leukemic blasts from G450 and K00339 expressed CD34 (Figure 1, G-I), whereas only about $1 \%$ of blasts were
CD34+ in G374. However, a leukemic cell line derived from G374 expressed CD34 (see Figure 3C).

Taken together, we observed the development of leukemia in 3 animals. In all 3 cases the leukemia arose in HOXB4GFP-expressing cells but not in YFP-expressing cells, suggesting that HOXB4 is the major contributor to the leukemia $(P=0.05)$.

Gammaretrovirus integration induced mutagenesis is associated with leukemogenesis. To examine the clonality of the leukemia, we performed Southern blot analyses. In G374, we detected 2 bands with the same intensity, suggesting 1 clone with 2 integration sites (Figure 2A). Analysis of DNA samples from different time points after transplantation confirmed this result (Supplemental Figure 5). In G450, we detected 1 band, demonstrating 1 clone with 1 integration site. DNA from K00339 showed 5 bands with the same intensity, suggesting 1 predominant clone with 5 inte- 
A

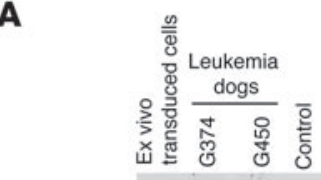

HOXB4
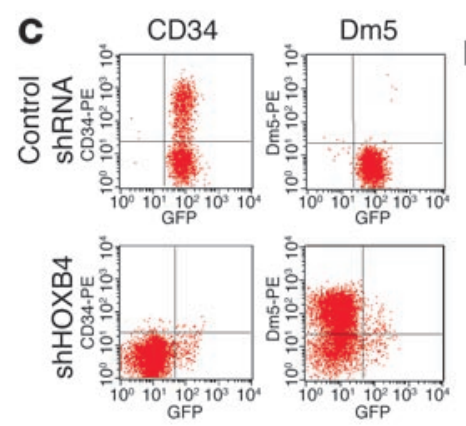
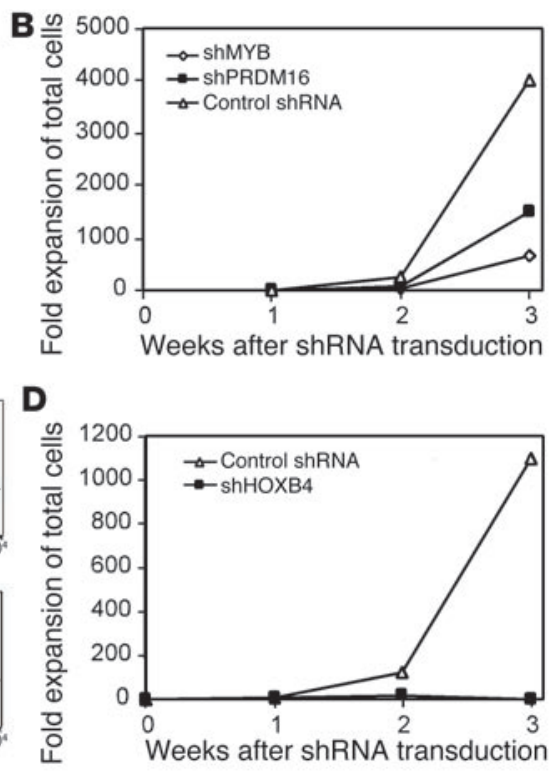

Figure 3

HOXB4 overexpression contributes to leukemogenesis. (A) Western blot analysis showed strong HOXB4 expression in marrow cells from leukemic animals. HOXB4-overexpressing dog cells in ex vivo culture were used as a positive control and marrow cells from normal dog as a negative control. (B) Knockdown of PRDM16 or MYB decreased the proliferation rate of the dog G374 leukemic cells. (C) HOXB4 knockdown in the leukemic cell line induced cell differentiation and (D) abolished cell proliferation. The stem cell marker CD34 and mature granulocyte marker Dm5 were used to evaluate cell differentiation. The leukemic cell line was cultured in medium supplemented with cSCF, thrombopoietin, and Flt3-L each at $100 \mathrm{ng} / \mathrm{ml}$. gration sites. PCR analysis of each integrant using DNA from a single colony derived from K00339 bone marrow confirmed this interpretation (data not shown).

Integration sites were determined by LAM-PCR (22). In dog $\mathrm{G} 374$, the vector was integrated at $100 \mathrm{~kb}$ upstream of the $c-m y b$ transcription start site (TSS) in the reverse orientation, and a second provirus was integrated into intron 3 of PR domain containing 16 (PRDM16) in the same orientation (Figure 2B). In G450, the vector was integrated at $90 \mathrm{~kb}$ downstream of ZFP36L2 TSS (Figure 2C). Of interest, we also observed 1 integration site at PRDM16 intron in K00339 (Figure 2D). Two integrants in this animal were at introns of single-stranded DNA binding protein 2 (SSBP2) and nonstructural maintenance of chromosomes element 1 homolog (NSMCE1) in reverse orientation, and 2 other integrants were at $640 \mathrm{bp}$ upstream of mago-nashi homolog 2 (MAGOH2) and $60 \mathrm{~kb}$ downstream of SOCS1 TSS (Figure 2D).

We next explored whether vector integration at oncogenes resulted in altered oncogene expression using real-time RT-PCR. In marrow from G374, expression of PRDM16 was detected, whereas in normal control samples PRDM16 was undetectable (Figure 2E). Further analysis demonstrated that the $5^{\prime}$ LTR was spliced into exon 4, activating the short isoform PRDM16 (Supplemental Figure 6), which lacks the PR domain and has been reported to play an important role in leukemogenesis (23). In addition, MYB expression was also upregulated.

In G450, integration at the ZFP36L2 locus was associated with increased expression of ZFP36L2 and thyroid adenoma associated (THADA) expression compared with control bone marrow cells (Figure 2F).

\section{Figure 4}

Increase in relative marking of leukemic clones over time. Real-time PCR was performed for dogs G374 (A) and G450 (B) and macaque K00339 (C) using LTR- and chromosome-specific primers. Shown are relative values that were normalized to $\beta$-actin or GAPDH, which quantify total genomic DNA. Standard curves were generated using a series of 8 dilutions of necropsy marrow DNA samples from each animal. PB, peripheral blood.
In K00339 bone marrow, we detected increased expression of PRDM16 and SOCS1 and decreased expression of SSBP2, a tumor suppressor (Figure 2G). Given that SOCS1 expression has been reported to be downregulated in many leukemic cells (24), it may suggest that the integration site at the SOCS1 locus has not contributed to leukemia. Expression of NSMCE1 was undetectable in all the samples, suggesting that it is a "passenger" integration site. Virus integration upstream of the TSS also appeared to activate MAGOH2 expression; however, its function in mammalian cells is largely unknown. Taken together, PRDM16 upregulation and SSBP2 downregulation, among other dysregulated genes, likely contributed to the development of leukemia in this animal.
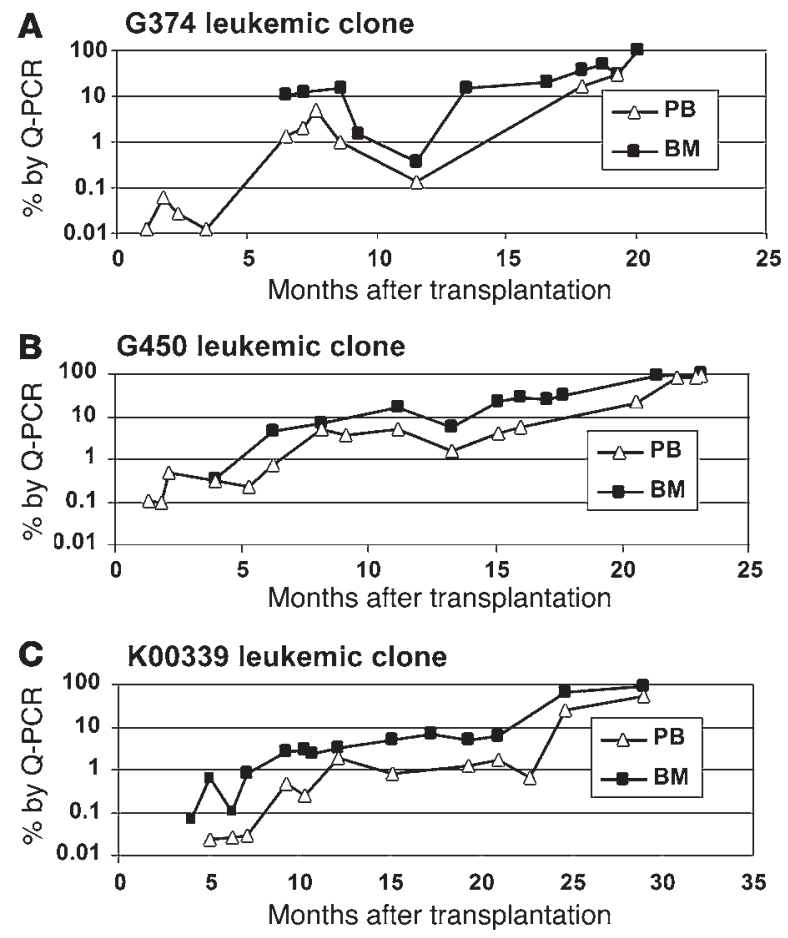
A

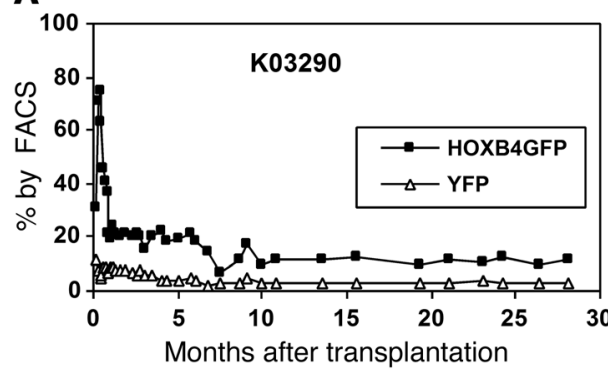

B

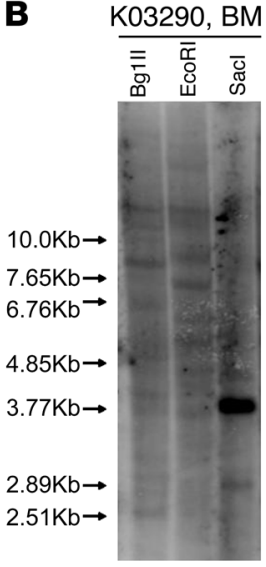

\section{Figure 5}

Characterization of macaque K03290, an animal with normal hematopoiesis. (A) Marking levels of K03290 in peripheral blood, which had been stabilized for more than 2 years. (B) Southern blot analysis showed multiple bands of HOXB4-overexpressing marrow cells. Digestion with Sacl, which cut the transgene twice, showed a 3.9-kb band for all the integrants. Bglll and EcoRI cut the transgene once, and so each band indicates an individual integrant.
HOXB4 overexpression is responsible for leukemogenesis. Flow cytometric analysis showed high-level expression of HOXB4GFP in these animals, which was confirmed by western blot (Figure 3A). To further explore the mechanisms underlying leukemogenesis, we established a leukemic cell line from G374 bone marrow; this line grows robustly in the presence of cytokines and expresses CD34. We designed shRNAs to PRDM16, MYB, and HOXB4 to determine whether the increased expression of these genes has contributed to leukemogenesis. As shown in Figure 3B, downregulation of PRDM16 and MYB expression only decreased the cell proliferation rate during a 3 -week culture period without a change in the percentage of $\mathrm{CD} 34^{+}$cells and no differentiation. In contrast, downregulation of HOXB4 resulted in the complete disappearance of $\mathrm{CD} 34^{+}$cells, and most cells expressed the mature granulocyte marker Dm5 after 2 weeks (Figure 3C). These findings demonstrate that HOXB4 knockdown resulted in the differentiation of the leukemic cells. Furthermore, differentiated cells stopped growing after 3 weeks. These data demonstrate the ability of HOXB4 in concert with key cooperating genes to promote stem cell self-renewal and inhibit cell differentiation in leukemic clones, thus eventually leading to overt leukemia.

Taken together, these data suggest that HOXB4 played a pivotal role in leukemogenesis through its ability to effectively collaborate with cooperating mutagenic events.

Dynamics of leukemic clones. We used SYBR Green real-time PCR to track and quantitate the leukemic clones in the animals (Figure 4, A-C). Between 1 and 2 months after transplantation, only $0.01 \%-0.1 \%$ of peripheral blood cells were leukemic clones. By 6 to 12 months, the contributions increased to about $1 \%$ in peripheral blood and up to $10 \%$ in marrow cells. However, there was no significant change over the subsequent year. Interestingly, the leukemic clone in G374 marrow increased to $10 \%$ at 6 months after transplantation but decreased to less than $1 \%$ at 11 months after transplantation. These data are consistent with the flow cytometric results (Figure 1A and data not shown) and Southern blot analysis (Supplemental Figure 5).

The latency of about 1 year before the development of overt leukemia in all animals suggests that more mutations may have accumulated in that time period, leading to the development of leukemic blasts. Karyotype analysis of marrow cells from the 3 leukemic animals, however, did not detect any gross abnormalities (data not shown). This does not rule out the possibility of gene deletions and duplications and accumulation of random nucleotide mutations that would not have been detected by standard karyotype analysis.

Characterization of another macaque with normal hematopoiesis. One (K03290) of 2 macaques that have been followed for more than 28 months has not shown any evidence of abnormalities thus far. The marking levels in peripheral granulocytes have stabilized at $10 \%$ for HOXB4 and 2\% for YFP (Figure 5A). Southern blot analysis of DNA from $\mathrm{HOXB} 4 \mathrm{GFP}^{+}$marrow cells revealed multiple bands, indicative of polyclonal hematopoiesis (Figure 5B). LAM-PCR analysis of $\mathrm{HOXB}_{4} \mathrm{GFP}^{+}$cells showed 9 integration sites (Table 1), among which mitochondrial ribosomal protein S23 (MRPS23), CD9, MMP8, and IGFBP8 have been reported to be involved in tumorigenesis. Thus, even with integration sites at oncogene loci, this macaque has not developed malignancy yet.

No evidence of abnormalities in animals that received MGMT-transduced cells. In contrast to the 3 of 4 animals that developed leukemia after transplantation of HOXB4-transduced cells, none of the more than 40 dogs and monkeys transplanted with transduced cells using transgenes other than HOXB4 and followed for an average of 3 years with marking levels greater than $1 \%$ have developed leukemia $(P<0.001)$. Among these animals were 5 dogs that received the MGMTGFP-transduced cells using the same murine stem cell virus-based (MSCV-based) retroviral vector as was used for the HOXB4 studies. These animals also received multiple rounds of chemotherapy after transplantation

\section{Table 1}

Integration sites retrieved from HOXB4-overexpressing cells in K03290

$\begin{array}{lcc}\text { Gene } & \text { Chromosome } & \text { Location } \\ \text { MRPS23 exon reverse } & 16 & 42076270 \\ \text { CD9 intron reverse } & 11 & 6340380 \\ \text { MMP8 before TSS } & 14 & 101345377 \\ \text { IGFBP7 before TSS } & 5 & 72212336 \\ \text { MYH9 } & 10 & 80333233 \\ \text { DYN } & 18 & 42102723 \\ \text { RUFTY2 } & 2 & 135082832 \\ \text { LMNB1 } & 6 & 123152116 \\ \text { Predicted gene before TSS } & 15 & 98031113\end{array}$

Several viral vector integration sites at known oncogene loci are identified. 

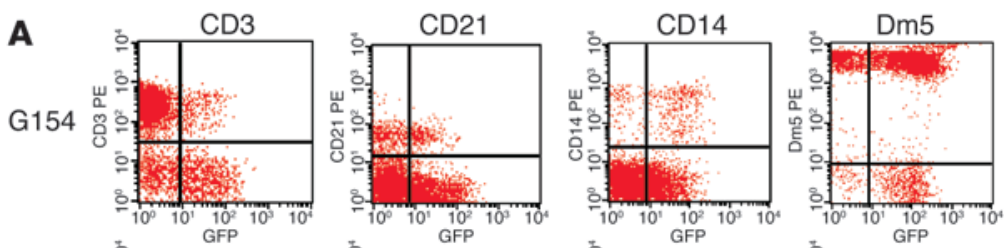

G179
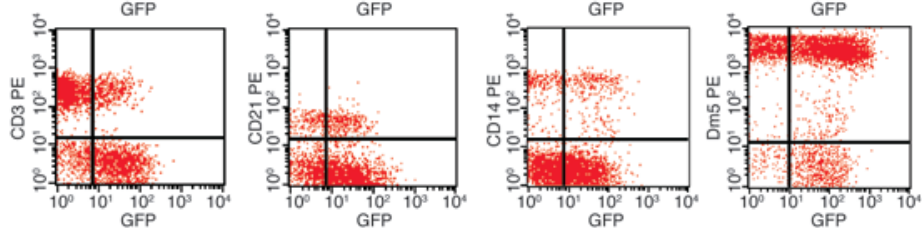

B
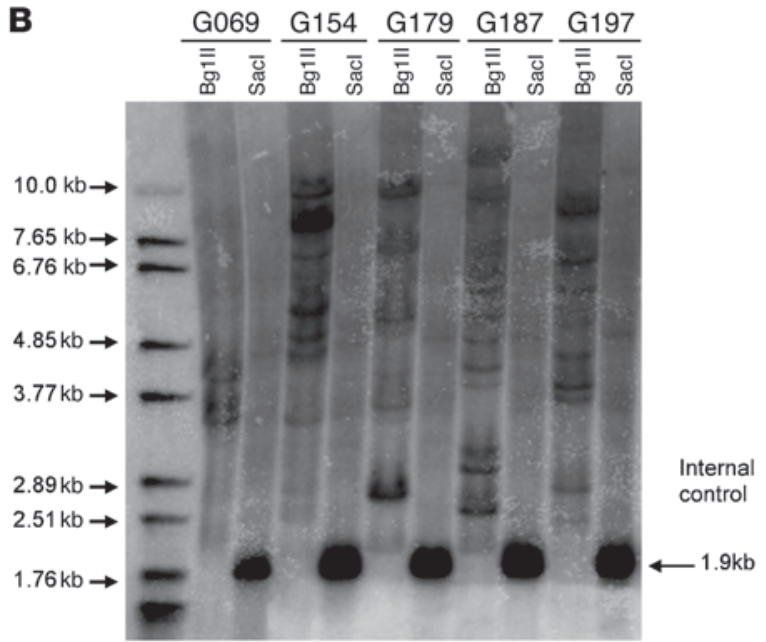

to increase the marking levels $(25,26)$. Five years after transplantation, the marking stabilized at high levels, in particular for granulocytes (Table 2). Peripheral blood and marrow analysis showed normal hematopoiesis with no evidence of leukemia (Figure 6A). Furthermore, Southern blot analysis of bone marrow DNA from these animals showed multiple bands, demonstrating polyclonality (Figure 6B).

Integration site analysis of the 5 animals and many other longterm follow-up animals has been published elsewhere $(22,27)$. Interestingly, we detected virus insertions at similar genes, including CCDN3, JAK1, LMO2, MYH9, and PRDM16, without the development of leukemia, further supporting the hypothesis that integrations at oncogene loci alone may not be sufficient to initiate leukemogenesis.

\section{Discussion}

Here, we report that retrovirus-mediated HOXB4 expression in hematopoietic repopulating cells led to monoclonality and leukemia in 2 out of 2 dogs and 1 out of 2 macaques, approximately 2 years after transplantation. In contrast, more than 40 dogs and monkeys received retrovirus-modified $\mathrm{CD} 34^{+}$cells with transgenes other than HOXB4, including 5 dogs that received MGMT-transduced cells using the same vector backbone as in the HOXB4 animals described here, and all of these animals have maintained normal polyclonal hematopoiesis after transplantation. A role of HOXB4 in the development of leukemia in these animals is further supported by HOXB4 knockdown experiments showing the differentiation of leukemic cells without HOXB4 overexpression.

\section{Figure 6}

Normal hematopoiesis in dogs that received MGMTtransduced cells using MSCV retroviral vector. (A) Subset analysis shows marked cells in all the lineages of blood cells. Analysis of peripheral blood from 2 representative animals is presented. (B) Southern blot analysis of marrow from 5 animals demonstrates polyclonality. Digestion with Bglll, which cuts vector once, releases a unique band for each integration sites, while digestion with Sacl, which cuts vector twice, releases a single internal control band of $1.9 \mathrm{~kb}$.

Retroviruses have long been known to cause leukemia by insertional mutagenesis. Clonal dominance of hematopoietic stem cells by retroviral gene marking has been observed in a large group of mice $(28,29)$; furthermore, high-copy retroviral gene transfer of marker or drug resistance gene induced leukemia in mice due to combinatorial insertional mutagenesis $(30,31)$. Studies in nonhuman primates using retroviral vector preparations containing replication-competent viruses induced T cell lymphoma (32). Using replication-incompetent viruses, there has only been 1 report, by Seggewiss et al. (33), of insertional mutagenesis in nonhuman primates. The investigators found a fatal myeloid sarcoma in 1 monkey 5 years after transplantation with a MSCV-based RD114pseudotyped retroviral vector. Interestingly this animal had repopulated with only 1 clone and had also received 1 cycle of chemotherapy. This animal was 1 of 7 animals that underwent transplantation using a similar vector and was followed for more than 5 years. These investigators also point out that this was the only animal that developed leukemia or abnormal hematopoiesis out of more than 80 large animals that have been followed long-term (33). These observations in large animals, together with clinical stem cell gene therapy trials for diseases other than SCID-X1, suggest that the development of leukemia induced by insertional mutagenesis alone occurs at very low incidence in large animals or humans, while gene therapy with a retroviral vector that expresses HOXB4 or IL2R $\gamma$ and possibly other growth-promoting genes significantly increases the incidence of leukemogenesis.

In our study, 3 of 4 animals that received HOXB4-overexpressing cells and had a follow-up of at least 2 years developed leu-

\section{Table 2}

Stable, high-level gene marking in granulocytes in dogs transplanted with MGMTGFP-transduced repopulating cells

\begin{tabular}{lcc} 
Animal & Follow-up (yr) & Marking in granulocytes (\%) \\
G069 & 5.6 & 38 \\
G154 & 5.2 & 96 \\
G179 & 5.1 & 91 \\
G187 & 4.9 & 94 \\
G197 & 4.8 & 76 \\
Mean & 5.1 & 79 \\
\hline
\end{tabular}

All animals received multiple cycles of chemotherapy for in vivo selection of gene-modified cells. 
kemia, a higher incidence than in the SCID-X1 trials. This may be because HOXB4 promotes stem cell self-renewal and extensive expansion of myeloid progenitors (34), while IL2R $\gamma$ expands only lymphoid cells. All 4 cases in the SCID-X1 trials were T cell leukemias, while all 3 leukemias in our study were myeloid leukemias, which is unlikely to be random coincidence. We would assume that there is no difference between these viral vectors in the initial selection of integration sites. Instead, our data suggest that it is a consequence of oncogene collaboration (or integration site collaboration), as suggested in a mouse study in which cooperativity between LMO2 and IL2R $\gamma$ was documented (35). Studies in our other animals have identified integration sites at LMO2 (22). However, we did not detect any integrations in sites at LMO2 in the gene-modified cells of the animals reported here, although we can not rule out that this occurred in minor clones but at levels below detection and without apparent consequence, perhaps due to HOXB4's reported ability to moderately suppress lymphoid repopulation $(36,37)$. Based on the activation of nearby genes known to be involved in leukemogenesis, the integration sites observed in the leukemic clones are very likely a consequence of sustained in vivo proliferation and selection in HOXB4-transduced repopulating cells.

Both dogs and monkeys developed leukemia approximately 2 years after transplantation. The number of integrations associated with the canine leukemias (1 or 2 integrations) was lower than in the monkey (5 integrations). In our previous studies we observed that HOXB4 expression levels were higher in dog cells than in nonhuman primate cells and that HOXB4 overexpression allowed for immortalization of dog cells but not monkey cells (34), which would support a more pronounced effect of HOXB4 in dog cells. These findings suggest that dogs may be more susceptible to leukemic transformation by HOXB4 (and perhaps other growth-promoting genes) and retroviral insertional mutagenesis. This possibility further suggests that the canine model may prove more sensitive for preclinical safety testing of candidate retroviral gene therapy vectors.

Clone-tracking PCR analysis demonstrated a substantial clonal expansion during early engraftment followed by a dormant phase lasting about 1 year. Growing evidence suggests that oncogenes induce DNA hyperreplication and DNA damage and that unrepaired or misrepaired damage eventually leads to clones capable of uncontrolled expansion $(38,39)$. In addition, a cancer mutator strain theory proposes that clones with hypermutations are associated with tumorigenesis (40). Thus, we propose that oncogene activation may increase mutation rates and accumulation of malignant clones, eventually resulting in malignancies.

Interestingly, HOXB4 overexpression has not been associated with leukemia in mice, although several other wild-type HOXA9 $(20,41,42)$ or mutant Hox fusions with NUP98 (42) are clearly leukemogenic. This may reflect differential potency of HOXB4 and other Hox transcription factors to have an impact on selfrenewal versus differentiation in different species. We also speculate that the much larger cell dose used for transplantation in these large animal models and the attendant larger number of integration sites (e.g., here we transplanted some 1,000-fold more retroviral integration sites compared with the mouse studies) resulted in an increased chance of insertional mutagenesis. The increased time of follow-up - here, animals were followed for over a year, compared with the usually shorter times for mice - may also allow detection of long latency leukemias in larger animals. Together, these findings highlight the importance of using large animal models for preclinical safety testing of gene therapy approaches involving genetic manipulation of primitive hematopoietic cells.

The 5 animals that received MGMT-expressing cells showed normal hematopoiesis even at 5 years after transplantation and with similar integration sites at oncogene loci. Our observations emphasize that the benefits of stem cell gene therapy can outweigh its potential risks in cases like MGMT-based gene therapy, where transgene-expressing cells do not possess an intrinsic growth advantage over control cells.

In conclusion, our findings in 2 clinically relevant large animal models suggest that transgenes capable of expanding hematopoietic repopulating cells may pose an increased risk of leukemogenesis relative to transgenes that do not confer an intrinsic constitutive selective advantage. Thus, our data suggest the continued need for caution in genetic manipulation of repopulating cells, particularly when the transgene may confer an intrinsic selective growth advantage. In addition, large animal studies, in particular dog studies, should be highly valuable models in assessing safety of retroviral vectors.

\section{Methods}

Animals. Nonhuman primates (Macaca nemestrina) were housed in the University of Washington National Primate Center, and dogs were housed at the Animal Health Resources unit of the Fred Hutchinson Cancer Research Center. All animal experiments and manipulations conducted were previously approved by the IACUCs of the Fred Hutchinson Cancer Research Center and the University of Washington.

Transplantation of HOXB4-transduced cells. Equal aliquots of MACS-purified dog CD34+ cells were transduced with Phoenix RD114 pseudotyped gammaretroviral vector MSCV-HOXB4-ires-GFP or control MSCV-ires-YFP after 2 days of prestimulation in Iscove's modified Dulbecco's medium (IMDM) supplemented with $12.5 \%$ horse serum, $12.5 \%$ fetal bovine serum, $10^{-6} \mathrm{M}$ hydrocortisone, $10^{-4} \mathrm{M} \beta$-mercaptoethanol, $2 \mathrm{mM}$ glutamine, $1 \%$ penicillin/streptomycin $(\times 100$ liquid; Invitrogen $)$ in the presence of Flt3-L, canine SCF, and canine G-CSF, each at $50 \mathrm{ng} / \mathrm{ml}$. For transduction, cells were exposed to retroviral vectors at an MOI of 1-2 for 4 hours in the presence of growth factors. After overnight culture in media containing growth factors, cells were re-exposed to the same MOI of concentrated vector for 4 hours. Immediately after this second exposure, cells were washed and infused into lethally irradiated animal (920 cGy). The transduction and transplantation of macaque cells have been previously reported (21).

FACS. Flow cytometry was used to determine the marking levels of the animals after transplantation. Blood samples from control animals that do not express GFP were used for gating. For subset analysis of dog cells, CD3, CD21, CD14, CD34, and Dm5 antibodies were used. For subset analysis of macaque cells, CD3, CD20, CD14, CD13, and CD34 antibodies were used.

Morphology. Bone marrow biopsy or necropsy smears were prepared for morphological examination. Glass slides were stained with WrightGiemsa stain. Digital pictures were taken with a Nikon microscope. Pathologists performed necropsy analysis of tissue samples from the dogs and monkey.

Southern blot analysis. Southern blot was performed to determine clonality. DNA from the marrow of animals was extracted using a Puregene DNA Purification Kit (Gentra Systems). DNA $(20 \mu \mathrm{g})$ was digested overnight with BglII, EcoRI, or SacI and electrophoresed on $0.8 \%$ agarose gel. Denatured DNA was transferred to $\mathrm{N}^{+}$nylon membrane and hybridized in QuickHyb solution (Amersham) with ${ }^{32} \mathrm{P}-$-labeled ires-GFP, which was cut from 
the MSCV-HOXB4-ires-GFP plasmid. Hybridization signals were detected using a Typhoon Phosphor Imaging System (Amersham Biosciences).

Integration site analysis. Integration site analysis was performed using LAM-PCR as described previously (22). The PCR products were cloned into TOPO vector for sequencing. The retrieved sequences were aligned with dog genome assembly May 2005 or rhesus macaque genome assembly January 2006 at the University of California Santa Cruz (UCSC) Genome Browser Web site (http://genome.ucsc.edu/cgi-bin/hgBlat?command=star t\&org=Dog [for dogs] and http://genome.ucsc.edu/cgi-bin/hgBlat?comma nd=start\&org=Rhesus [for rhesus]) to determine the integration sites.

Knockdown experiments. A leukemic cell line was derived from the marrow of dog G374 at necropsy. Cells were cultured in IMDM supplemented in $12.5 \%$ horse serum and $12.5 \%$ fetal bovine serum in the presence of canine SCF, thrombopoietin, and Flt3-L, each at $100 \mathrm{ng} / \mathrm{ml}$. shRNA constructs were cloned into an LMP vector (Open Biosystems) that coexpressed puro ${ }^{\mathrm{r}}$ gene. Downregulation of the targeted genes was confirmed by real-time PCR. Phoenix RD114 pseudotyped viral vectors were produced by transient transfection of $293 \mathrm{~T}$ cells. The leukemic cells were transduced with viral vectors and selected with $5 \mu \mathrm{g} / \mathrm{ml}$ puromycin the next day. Selected cells were kept in culture or harvested for RNA extraction and real-time RT-PCR analysis.

Real-time RT-PCR. Total RNA was extracted using RNeasy Mini Kit (Qiagen). Residual DNA was eliminated by DNase I treatment. Canine or macaque mRNA sequences were determined by aligning human RNA sequences with canine or macaque genome sequences at the UCSC Genome Browser Web site (http://genome.ucsc.edu/). Reverse transcription was performed using ThermoScript RT-PCR System (Invitrogen). Primers were designed with Primer 3.0 program. For quantitative analysis of gene expression, SYBR Green real-time PCR was performed on 7500 Real Time PCR System (Applied Biosystems). The data were normalized to GAPDH for monkey samples or to $\beta$-actin for dog samples.
Clone tracking real-time PCR. Two primers, one on MSCV transgene and another on genome sequence of the integration sites, were designed to track the contribution of the leukemic clone to hematopoiesis. For SYBR Green real-time PCR, genomic DNA samples from marrow at necropsy were used for generation of the standard curve, assuming that all the $\mathrm{HOXB} 4 \mathrm{GFP}^{+}$cells were from the leukemic clone, which was supported by Southern blot data.

Western blot. Bone marrow cells from the leukemic animals and control normal animal were subjected to protein extraction and western blot analysis of HOXB4 expression, which has been detailed elsewhere (21).

Statistics. We analyzed the data by Fisher's exact test. $P$ values less than 0.05 were considered to be statistically significant.

\section{Acknowledgments}

We thank Bonnie Larson and Helen Crawford for help in preparing the manuscript. We thank James Fletcher and the University of Washington National Primate Research Center staff for the care of the monkeys and the Fred Hutchinson Cancer Research Center Dog Lab staff for the care of the dogs. We thank Barry Storer and Yi Cao for performing statistical analysis. This work was supported by NIH grants HL53750, HL36444, HL74162, HL84345, DK56465, and DK47754.

Received for publication October 31, 2007, and accepted in revised form February 6, 2008.

Address correspondence to: Hans-Peter Kiem, Fred Hutchinson Cancer Research Center, 1100 Fairview Ave. N., Mail Stop D1-100, PO Box 19024, Seattle, Washington 98109-1024, USA. Phone: (206) 667-4425; Fax: (206) 667-6124; E-mail: hkiem@fhcrc.org.
1. Verma, I.M., and Weitzman, M.D. 2005. Gene therapy: twenty-first century medicine. Annu. Rev. Biochem. 74:711-738.

2. Cavazzana-Calvo, M., et al. 2000. Gene therapy of human severe combined immunodeficiency (SCID)-X1 disease. Science. 288:669-672.

3. Hacein-Bey-Abina, S., et al. 2002. Sustained correction of $\mathrm{X}$-linked severe combined immunodeficiency by ex vivo gene therapy. N. Engl. J. Med. 346:1185-1193.

4. Schwarzwaelder, K., et al. 2007. Gammaretrovirusmediated correction of SCID-X1 is associated with skewed vector integration site distribution in vivo. J. Clin. Invest. 117:2241-2249.

5. Deichmann, A., et al. 2007. Vector integration is nonrandom and clustered and influences the fate of lymphopoiesis in SCID-X1 gene therapy. J. Clin. Invest. 117:2225-2232.

6. Gaspar, H.B., et al. 2006. Successful reconstitution of immunity in ADA-SCID by stem cell gene therapy following cessation of PEG-ADA and use of mild preconditioning. Mol. Ther 14:505-513.

7. Aiuti, A., et al. 2002. Correction of ADA-SCID by stem cell gene therapy combined with nonmyeloablative conditioning. Science. 296:2410-2413.

8. Aiuti, A., et al. 2007. Multilineage hematopoietic reconstitution without clonal selection in ADASCID patients treated with stem cell gene therapy. J. Clin. Invest. 117:2233-2240.

9. Ott, M.G., et al. 2006. Correction of X-linked chronic granulomatous disease by gene therapy, augmented by insertional activation of MDS1EVI1, PRDM16 or SETBP1. Nat. Med. 12:401-409.

10. Hacein-Bey-Abina, S., et al. 2003. LMO2-associated clonal $\mathrm{T}$ cell proliferation in two patients after gene therapy for SCID-X1 [erratum 2003, 302:568]. Science. 302:415-419.

11. Cavazzana-Calvo, M. and Fischer, A. 2007. Gene therapy for severe combined immunodeficiency: are we there yet? J. Clin. Invest. 117:1456-1465.

12. Baum, C. 2007. Insertional mutagenesis in gene therapy and stem cell biology. Curr. Opin. Hematol. 14:337-342.

13. Shou, Y., Ma, Z., Lu, T., and Sorrentino, B.P. 2006. Unique risk factors for insertional mutagenesis in a mouse model of XSCID gene therapy. Proc. Natl. Acad. Sci. U. S. A. 103:11730-11735.

14. Montini, E., et al. 2006. Hematopoietic stem cell gene transfer in a tumor-prone mouse model uncovers low genotoxicity of lentiviral vector integration. Nat. Biotechnol. 24:687-696.

15. Kiem, H.-P., et al. 2004. Long-term clinical and molecular follow-up of large animals receiving retrovirally transduced stem and progenitor cells: no progression to clonal hematopoiesis or leukemia. Mol. Ther. 9:389-395.

16. Antonchuk, J., Sauvageau, G., and Humphries, R.K. 2001. HOXB4 overexpression mediates very rapid stem cell regeneration and competitive hematopoietic repopulation. Exp. Hematol. 29:1125-1134.

17. Sauvageau, G., et al. 1995. Overexpression of HOXB4 in hematopoietic cells causes the selective expansion of more primitive populations in vitro and in vivo. Genes Dev. 9:1753-1765.

18. Antonchuk, J., Sauvageau, G., and Humphries, R.K. 2002. HOXB4-induced expansion of adult hematopoietic stem cells ex vivo. Cell. 109:39-45.

19. Krosl, J., et al. 2003. In vitro expansion of hematopoietic stem cells by recombinant TAT-HOXB4 protein. Nat. Med. 9:1428-1432.

20. Abramovich, C., Pineault, N., Ohta, H., and Humphries, R.K. 2005. Hox genes: from leukemia to hematopoietic stem cell expansion. Ann. N. Y. Acad. Sci. 1044:109-116.

21. Zhang, X.-B., et al. 2006. Differential effects of HOXB4 on nonhuman primate short- and long- term repopulating cells. PLoS Med. 3:e173.

22. Beard, B.C., et al. 2007. Comparison of HIV-derived lentiviral and MLV-based gammaretroviral vector integration sites in primate repopulating cells. Mol. Ther. 15:1356-1365.

23. Nishikata, I., et al. 2003. A novel EVI1 gene family, MEL1, lacking a PR domain (MEL1S) is expressed mainly in $\mathrm{t}(1 ; 3)(\mathrm{p} 36 ; \mathrm{q} 21)$-positive AML and blocks G-CSF-induced myeloid differentiation. Blood. 102:3323-3332.

24. Wormald, S., and Hilton, D.J. 2007. The negative regulatory roles of suppressor of cytokine signaling proteins in myeloid signaling pathways (Review). Curr. Opin. Hematol. 14:9-15.

25. Neff, T., et al. 2003. Methylguanine methyltransferase-mediated in vivo selection and chemoprotection of allogeneic stem cells in a large-animal model. J. Clin. Invest. 112:1581-1588.

26. Neff, T., et al. 2005. Polyclonal chemoprotection against temozolomide in a large-animal model of drug resistance gene therapy. Blood. 105:997-1002.

27. Beard, B.C., et al. 2007. Unique integration profiles in a canine model of long-term repopulating cells transduced with gammaretrovirus, lentivirus, and foamy virus. Hum. Gene Ther. 18:423-434.

28. Kustikova, O., et al. 2005. Clonal dominance of hematopoietic stem cells triggered by retroviral gene marking. Science. 308:1171-1174.

29. Kustikova, O.S., et al. 2007. Retroviral vector insertion sites associated with dominant hematopoietic clones mark "stemness" pathways. Blood. 109:1897-1907.

30. Bunting, K.D., Galipeau, J., Topham, D., Benaim, E., and Sorrentino, B.P. 1998. Transduction of murine bone marrow cells with an MDR1 vector enables ex vivo stem cell expansion, but these expanded grafts cause a myeloproliferative syndrome in transplanted mice. Blood. 92:2269-2279. 
31. Modlich, U., et al. 2005. Leukemias following retroviral transfer of multidrug resistance 1 (MDR1) are driven by combinatorial insertional mutagenesis. Blood. 105:4235-4246.

32. Donahue, R.E., et al. 1992. Helper virus induced $\mathrm{T}$ cell lymphoma in nonhuman primates after retroviral mediated gene transfer. J. Exp. Med. 176:1125-1135.

33. Seggewiss, R., et al. 2006. Acute myeloid leukemia is associated with retroviral gene transfer to hematopoietic progenitor cells in a rhesus macaque. Blood. 107:3865-3867.

34. Zhang, X.-B., Schwartz, J.L., Humphries, R.K., and Kiem, H.-P. 2007. Effects of HOXB4 overexpression on ex vivo expansion and immortalization of hematopoietic cells from different species. Stem Cells. 25:2074-2081.

35. Dave, U.P., Jenkins, N.A., and Copeland, N.G. 2004. Gene therapy insertional mutagenesis insights. Science. 303:333.

36. Schiedlmeier, B., et al. 2003. High-level ectopic HOXB4 expression confers a profound in vivo competitive growth advantage on human cord blood CD34+ cells, but impairs lymphomyeloid differentiation. Blood. 101:1759-1768.

37. Pilat, S., et al. 2005. HOXB4 enforces equivalent fates of ES-cell-derived and adult hematopoietic cells. Proc. Natl. Acad. Sci. U. S. A. 102:12101-12106. 38. Di Micco, R., et al. 2006. Oncogene-induced senescence is a DNA damage response triggered by DNA hyper-replication. Nature. 444:638-642.

39. Bartkova, J., et al. 2006. Oncogene-induced senescence is part of the tumorigenesis barrier imposed by DNA damage checkpoints. Nature. 444:633-637.

40. Bielas, J.H., Loeb, K.R., Rubin, B.P., True, L.D., and Loeb, L.A. 2006. Human cancers express a mutator phenotype. Proc. Natl. Acad. Sci. U. S. A. 103:18238-18242.

41. Buske, C., and Humphries, R.K. 2000. Homeobox genes in leukemogenesis. Int. J. Hematol. 71:301-308.

42. Pineault, N., Abramovich, C., Ohta, H., and Humphries, R.K. 2004. Differential and common leukemogenic potentials of multiple NUP98-Hox fusion proteins alone or with Meis1. Mol. Cell. Biol. 24:1907-1917. 